Reprod. Nutr. Dévelop., 1980, 20 (4 B), 1267-1271.

\title{
Effet d'un excès de sels biliaires dans la lumière duodénale sur l'équipement enzymatique du tissu pancréatique chez le Porc
}

\author{
par J. MOUROT, T. CORRING, M. CHARLES *
}

avec la collaboration technique de Anne-Marie GUEUGNEAU, Georgette FREDOU, A. ROGER et A. VAN HECKE

Laboratoire de Physiologie de la Nutrition, I.N.R.A, 78350 jouy en Josas

* C. N. R. S.-C. B. B. M., 31, chemin Joseph-Aiguier, 13274 Marseille cedex 2

Summary. Effect of an 8-day intraduodenal bile salts load on the enzyme equipment of pig pancreatic fissue.

This study reports the effect of long-term (8 days) addition of bile salts in the duodenal lumen of the pig in which the bile enterohepatic cycle was fully maintained. Seventeen pigs were fitted with permanent fistulae in the bile duct and the duodenum. The bile collected in 9 pigs was returned to the intestine, while that of the other 8 pigs was returned after a 30 p. 100 excess in bile salts, supplied by lyophilized porcine bile, had been added.

At the end of the 8-day experimental period, the enzymes were determined in the fresh total pancreatic glands of all the pigs. The results showed no significant differences in lipase, colipase, amylase, chymotrypsin or trypsin activities in either group of animals.

The existence of an in vivo relationship between bile salts and colipase secretions is hypothesized.

Il a été démontré in vitro qu'il existe dans le processus de l'hydrolyse des triglycérides alimentaires un antagonisme d'action entre sels biliaires et colipase pancréatique. Cette dernière lève l'inhibition exercée par les sels biliaires sur l'activité enzymatique de la lipase pancréatique (Borgström, 1977 ; Rietsch et al., 1977 ; Verger, Rietsch et Desnuelle, 1977).

Dans le but de vérifier si cet antagonisme se traduit par l'existence d'une relation in vivo entre sécrétion biliaire et sécrétion colipasique une première expérience a montré que l'absence prolongée de bile dans la lumière intestinale ( 8 jours) ne modifie pas les teneurs tissulaires des enzymes et de la colipase pancréatiques (Corring ef al., 1979). De plus une étude récente (Mourot et al., données non publiées) montre chez le Porc que la dérivation de la sécrétion biliaire (jusqu'à 48 h) n'entraîne aucune modification de la composition en enzymes et en colipase du suc pancréatique recueilli sur le même animal.

Le travail rapporté ci-après a pour but l'étude de l'effet d'un excès de sels biliaires dans la lumière duodénale sur le niveau des enzymes et de la colipase du tissu pancréatique du Porc. 


\section{Matériel et méthodes.}

Animaux et régimes. - Dix-sepł porcs mâles castrés, de race Large White et de poids vif moyen de $47 \pm 4 \mathrm{~kg}$ en début d'expérience, ont été utilisés. Tous les animaux ont été munis de fistules permanentes du bas cholédoque et du duodénum, selon la technique décrite dans un précédent travail (Juste et al., 1979). Les animaux reçoivent un régime à base de céréales à 16-17 p. 100 de protéines ef 2 p. 100 de lipides, identique à celui utilisé précédemment (Corring et al., 1979). Ce régime est distribué en 2 repas de $800 \mathrm{~g}$ chacun par jour (poids aliments/poids eau : 1/2).

Schéma expérimental. - Après fistulation, les animaux sont placés en cage individuelle, du type cage à métabolisme. Après une semaine de rétablissement postopératoire, ils sont soumis au schéma expérimental suivant :

1) Première période expérimentale de 8 jours : collecte de la bile excrétée sur chaque porc avec mesures des volumes recueillis et prélèvements d'échantillons à $9 \mathrm{~h}$ et $16 \mathrm{~h}$ en vue de la détermination de la teneur en sels biliaires totaux (Hurlock ef Talalay, 1957). Au cours de cette période on obtient, pour chaque animal, une concentration moyenne quotidienne et une excrétion de sels biliaires moyenne quotidienne.

2) Deuxième période expérimentale de 8 jours. Les porcs sont répartis en 2 lots : un lot témoin de 9 animaux chez qui la bile collectée est retournée au niveau duodénal ef un lot expérimental de 8 animaux chez qui la bile collectée est réintroduite dans la lumière duodénale après addition d'un lyophilisat de bile provenant de porcs fistulés hors expérience. La quantité de lyophilisat ajouté a été déterminée d'après sa teneur en sels biliaires tołaux et correspond à un excès de 30 p. 100 de sels biliaires par rapport à l'excrétion moyenne quotidienne de chaque animal expérimental, déterminée pendant la première période expérimentale.

Dosages. - Immédiatement après abattage, le pancréas entier est prélevé, pesé et broyé à $0{ }^{\circ} \mathrm{C}$ dans de l'eau distillée $(6,5 \mathrm{ml} / \mathrm{g}$ de pancréas frais). Les protéines totales et les activités enzymatiques de la chymotrypsine, de la trypsine, de l'amylase, de la lipase et de la colipase ont été déterminées dans les homogénats pancréatiques selon des méthodes citées précédemment (Corring et al., 1979). En ce qui concerne le dosage de la lipase, son activité a d'abord été déterminée à $\mathrm{pH} \mathrm{9,0} \mathrm{sur} \mathrm{substrat} \mathrm{trio-}$ léate en présence de sels biliaires purifiés (concentration finale : $6 \mathrm{mM}$ ) ; cette activité, appelée résiduelle ou partielle, représente l'activité dépendante de la colipase tissulaire. La même réaction a été suivie après saturation de la lipase par de la colipase préparée par ailleurs (à partir de tissu frais de pancréas de porc, précipitation à $\mathrm{pH} 2$, surnageant de centrifugation lyophilisé). Cette activité, appelée potentielle ou totale, est utilisée pour estimer le degré de saturation en colipase de la lipase résiduelle. Les résultats concernant l'activité de la lipase sont exprimés en activité potentielle.

\section{Résultats.}

Le poids relatif du pancréas (par $\mathrm{kg}$ de poids vif), la teneur en protéines totales du tissu pancréatique et les activités enzymatiques spécifiques (exprimées 
par $\mathrm{mg}$ de protéines totales) pour les 2 lots d'animaux sont rapportés dans le fableau 1.

\section{TABLEAU 1}

Activités enzymatiques ef colipasique dans le fissu pancréatique du porc après surcharge intraduodénale de sels biliaires pendant 8 jours

\begin{tabular}{|c|c|c|c|}
\hline & Lot témoin $\left({ }^{1}\right)$ & Lot expérimental $\left({ }^{2}\right)$ & $\begin{array}{l}\text { Signification } \\
\text { statistique }\end{array}$ \\
\hline 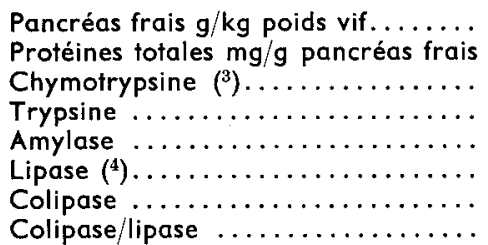 & $\begin{aligned} & 1,8 \pm 0,2 \\
& 204 \pm 20 \\
& 9,5 \pm 1,4 \\
& 1,96 \pm 0,5 \\
& 2160 \pm 410 \\
& 28,4 \pm 4,5 \\
& 43,6 \pm 1,8 \\
& 1,61 \pm 0,2\end{aligned}$ & $\begin{aligned} 2,0 & \pm 0,2 \\
202 & \pm 30 \\
9,3 & \pm 1,6 \\
2,10 & \pm 0,4 \\
2048 & \pm 400 \\
26,6 & \pm 4,1 \\
43,2 & \pm 6,6 \\
1,65 & \pm 0,2\end{aligned}$ & $\begin{array}{l}\text { NS } \\
\text { NS } \\
\text { NS } \\
\text { NS } \\
\text { NS } \\
\text { NS } \\
\text { NS } \\
\text { NS }\end{array}$ \\
\hline
\end{tabular}

(1) Animaux dont la bile a été retournée au niveau duodénal.

(2) Animaux dont la bile a été retournée au niveau duodénal après addition de sels biliaires.

$\left({ }^{3}\right)$ Toutes les activités enzymatiques sont exprimées en activités spécifiques (par mg de protéines totales pancréatiques).

(4) Lipase totale ou potentielle.

On constate, quel que soit le critère considéré, qu'il n'existe aucune différence significative entre les données obtenues chez les animaux témoins et les animaux ayant reçu un excès de sels biliaires dans la lumière intestinale.

\section{Discussion.}

II a été précédemment montré que l'absence de bile dans la lumière intestinale, qu'elle soit prolongée ( 8 jours) ou à plus court terme (jusqu'à $48 \mathrm{~h}$ ), n'affecte pas la composition enzymatique et colipasique du tissu (Corring et al., 1979) et du suc pancréatique (Mourot et al., données non publiées) chez le porc. Les résultats obtenus dans l'étude rapportée souligne que chez la même espèce, le recyclage entérohépatique étant intégralement conservé, une surcharge intra-intestinale de 30 p. 100 de sels biliaires pendant 8 jours ne conduit à aucune modification des teneurs en enzymes digestives et en colipase du tissu pancréatique.

Les études portant sur la relation in vivo entre sécrétion biliaire ef sécrétion pancréatique sont nombreuses mais aboutissent à des résultats contradictoires. Ainsi, l'addition de bile ou de sels biliaires dans la lumière intestinale stimule la sécrétion pancréatique totale chez l'Homme (Forell ef al., 1971 ; Osnes ef al., 1978) et le Chien (Konturek ef Thor, 1973). En revanche selon Thomas et Crider (1943) le volume pancréatique est inhibé chez cette dernière espèce. Lorsque la bile est éliminée de la lumière duodénale, la sécrétion du pancréas exocrine est stimulée chez le Rał (Kuroyanagi ef Necheles, 1962 ; Green et Nasset, 1977 ; Staub et Sarles, 1979). L'activité amylasique est par contre diminuée, également chez le Rat, selon Geratz et Lamb (1974). La compréhension de ces contradictions ne paraît pas facile compte tenu du 
nombre de facteurs pouvant être mis en cause : différence d'espèces, origine exogène de la bile ou des sels biliaires utilisés, méthodologie expérimentale en particulier dans l'étude de la sécrétion pancréatique. Il faut cependant constater que, quel que soit le sens de la variation, la présence ou l'absence de bile ou de sels biliaires affecte la composition de la sécrétion pancréatique chez toutes les espèces utilisées, sauf chez le Porc.

En ce qui concerne l'aspect particulier d'une relation in vivo entre sécrétion des sels biliaires ef sécrétion de colipase, il n'existe à notre connaissance aucune étude réalisée excepté celles chez le Porc dans notre laboratoire. Les résultats montrent l'absence d'une telle relation, que la bile soit dérivée (Corring ef al., 1979) ou que l'on pratique une surcharge en sels biliaires dans la lumière intestinale. Une première conclusion serait qu'il n'existe pas chez le Porc de relation physiologique entre les deux sécrétions. On rencontre des cas où des phénomènes démontrés in vitro ne sont ni confirmés, ni la traduction de processus physiologiques existant in vivo. Cependant, il paraît important de souligner que la méthodologie expérimentale sur animal fistulé ne peuł présenter les mêmes caractères de précision et de rigueur que ceux de l'expérimentation in vitro. Par ailleurs, on sait que le rapport colipase/lipase est chez le Porc plus élevé que chez les autres espèces étudiées (Julien ef al., 1979). On sait également que le niveau tissulaire de la colipase du Porc varie significativement sous l'effet d'une modification de la quantité de lipides ingérés (Mourot et Corring, 1979). Cela conduit à l'expression d'une deuxième conclusion sous forme d'hypothèse : chez le Porc, la colipase serait en excès tel que l'absence de bile cu la surcharge de sels biliaires dans la lumière intestinale ne peut être, comme la modification de la quantité de lipides ingérés, un facleur suffisant pour provoquer une variation sensible de sa teneur dans le tissu pancréatique. Dans le but de vérifier cette hypothèse, il serait intéressant de réaliser chez d'autres espèces telles que le Rat, les schémas expérimentaux que nous avons appliqués dans nos éłudes chez le Porc.

Journées Ingestion-Digestion-Absorption de l'Association fronçaise de Nutrition, Paris, 15-16 novembre 1979.

Remerciements. - Ce travail a été réalisé dans le cadre de l'action concertée DGRST 77.7.0472 — Interrelations Aliment — Tube Digestif.

\section{Références}

BORGSTRÖM B., 1977. The action of bile salts and other detergents on pancreatic lipase and the interaction with colipase. Biochim. biophys. Acta, 488, 381-391.

CORRING T., MOUROT J., CHARLES M., LÉGER C., 1979. Dérivation de la sécrétion biliaire ef activités enzymatiques dans le tissu pancréatique chez le porc. Ann. Biol. anim. Bioch. Biophys., 19, 833-836.

FORELL M. M., OTTE M., KOHL J. H., LEHNERT P., STAHLHEBER H. P., 1971. The influence of bile and pure bile salts in pancreatic secretion in man. Scand. J. Gastroenterol., 6, 262-266.

GERATZ J. D., LAMB IV J. C., 1974. Influence of bile duct ligation on exocrine pancreatic secretory activity in the rat. Am. J. Physiol., 277, 119-122.

GREEN G., NASSET E. S., 1977. Effect of bile duct obstruction on pancreatic enzyme secretion and intestinal proteolytic enzyme activity in the rat. Dig. Dis., 22, 437-444. 
HURLOCK B., TALALAY P., 1957. Principles of the enzymatic measurement of steroids. J. biol. Chem., 227, 37-52.

JULIEN R., RATHELOT J., CANIONI L., SARDA L., 1979. Etude comparée de l'effel de la colipase pancréatique sur l'activité des lipases de mammifères et de champignons. Ann. Biol. anim. Bioch. Biophys., 19, 819-823.

JUSTE C., CORRING T., BREANT P., 1979. Excrétion biliaire chez le porc : niveau et réponse au repas. Ann. Biol. anim. Bioch. Biophys., 19, 79-90.

KONTUREK S. J., THOR P., 1973. Effect of diversion and replacement of bile on pancreatic secretion. Dig. Dis. 18, 971-977.

KUROYANAGI Y., NECHELES H., 1962. Pancreatic secretion in the rat : effect of bile stasis and of bile salt. Am. J. Physiol., 203, 60-62.

MOUROT J., CORRING T., 1979. Adaptation of the lipase-colipase system to dietary lipid content in pig pancreatic tissue. Ann. Biol. anim. Bioch. Biophys., 19, 119-124.

OSNES M., HANSSEN L. E., FLATEN O., MYREN J., 1978. Exocrine pancreatic secretion and immunoreactive secretin (IRS) release after intraduodenal instillation of bile in man. Gut, 19, 180-184.

RIETSCH J., PATTUS F., DESNUELLE P., VERGER R., 1977. Further studies of mode of action of lipolytic enzymes. J. biol. Chem., 252, 4313-4318.

STAUB J. L., SARLES H., 1979 . Inhibition of Rat basal pancreatic secretion by intraduodenal bile. Digest. Dis. Sci., 24, 602-608.

THOMAS E. J., CRIDER J. C., 1943. The effect of bile in the intestine on the secretion of pancreatic juice. Am. J. Physiol., 138, 548-552.

VERGER R., RIETSCH J., DESNUELLE P., 1977. Effects of colipase on hydrolysis of monomolecular films by lipase. J. biol. Chem., 252, 4319-4325. 